\title{
Listagem da entomofauna antófila do estado de Mato Grosso do Sul, Brasil
}

\author{
Maria Rosângela Sigrisł ${ }^{1}$, Camila Aoki ${ }^{2}$, Camila Silveira de Souza ${ }^{3}$, Sebastião Laroca ${ }^{4}$, Jennifer Elaine \\ Maier', Márcia Rocha Vicente' , Fabrício Hiroiuki Oda ${ }^{5}$ \& Hélder Consolaro ${ }^{6}$
}

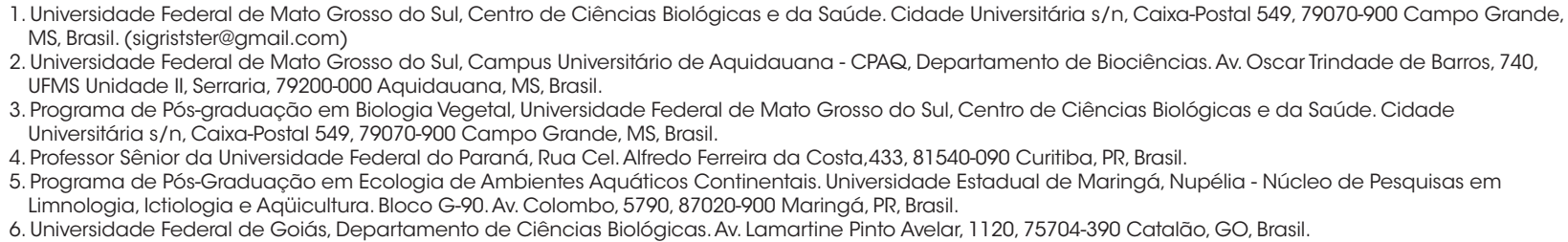

Recebido 25 novembro 2016

Aceito 6 fevereiro 2017

DOI: $10.1590 / 1678-4766 e 2017150$

\begin{abstract}
Checklist of anthophilous entomofauna from Mato Grosso do Sul state, Brazil. We present a checklist of the anthophilous entomofauna of Mato Grosso do Sul state (MS) based on information compiled from 17 studies, most of them achieved in Cerrado vegetation $(\mathrm{n}=11)$ and less frequently in the Pantanal $(n=6)$. We recorded 10 groups of insects, belonging to six orders, 80 families and 411 species. Higher richness was sampled for Cerrado (307 spp.) compared to Pantanal (147 spp.), with only 43 species (10.4\%) common to both biomes. Anthophila was the richest group (155 spp.), followed by beetles (82), butterflies/moths (53) and wasps (45), which totalized $81.5 \%$ of species. The highest richness of bees is related to the fact the most studies are concentrated in this group, besides the specialization of this group in utilization of floral resources. Surprising was the beetles occupying second place compared to others more "active" and "habitual" visitors, such as butterflies and flies. The entomofauna collections and research teams in MS are limited and/or incipient and they should be strenghtened through partnerships, exchange and capacity building in this biological group.
\end{abstract}

KEYWORDS. Insect biodiversity, floral visitor, BIOTA MS Program.

RESUMO. Apresentamos listagem da entomofauna visitante de flores do estado de Mato Grosso do Sul (MS) com base na compilação de informações obtidas em 17 estudos, a maioria realizado em áreas de Cerrado $(\mathrm{n}=11)$ e menos frequentemente no Pantanal $(\mathrm{n}=6)$. Foram registrados 10 grupos de insetos, pertencentes a seis ordens, 80 famílias e 411 espécies. Maior riqueza foi amostrada para o Cerrado (307 spp.) que Pantanal (147 spp.), com somente 43 espécies $(10,4 \%)$ em comum entre estes biomas. Anthophila foi o grupo mais rico (155 spp.), seguido por besouros (82), borboletas/mariposas (53) e vespas (45), que junto totalizaram $81,5 \%$ das espécies. Maior riqueza de abelhas está relacionada ao fato da maioria dos trabalhos enfocarem este grupo, além da especialização deste grupo na utilização de recursos florais. Surpreendente foi o segundo lugar ocupado por besouros em relação a outros visitantes mais "ativos" e "habituais", como lepidópteros e moscas. Em MS os acervos e grupos de pesquisa com entomofauna antófila são restritos e/ ou incipientes, sendo necessário fortalecimento dos mesmos através de parcerias, intercâmbios e formação de recursos humanos na área.

PALAVRAS-CHAVE. Biodiversidade de insetos, visitante floral, Programa BIOTA MS

A fauna antófila é constituída por diversos grupos de animais que visitam flores para buscar recursos alimentares (e.g. pólen, néctar, óleo, tecido floral, "sweet jelly"), materiais para a construção dos ninhos (óleo, resina) e/ou "perfumes" e podem também utilizar a flor como sítio de cópula, berçário e/ ou dormitório (SIMPSON \& NeFF, 1981; Proctor et al., 1996; Sazima et al., 2001; Stehlmann \& Semir, 2001; DufaŸ \& ANSTETT, 2003). Os visitantes florais são invertebrados (e.g. abelhas, moscas, besouros, vespas, borboletas, mariposas, grilos, baratas) e vertebrados (e.g. beija-flores, morcegos, lagartos) de várias Ordens (FAegri \& VAN DER PIJL, 1979; Proctor et al., 1996; NyHagen et al., 2001; VlasákovÁ et al., 2008; MichenEAU et al., 2010), com predomínio de insetos. A maioria dos insetos antófilos geralmente não é amostrada nos levantamentos faunísticos habituais, exceto abelhas, que são essencialmente herbívoras e dependentes dos recursos florais.

A fauna antófila quando visita as flores, principalmente as abelhas (Anthophila), pode atuar como polinizadores. A polinização é serviço ambiental de grande relevância para a manutenção da integridade dos ecossistemas e para a sustentabilidade da agricultura, necessitando haver maior atenção no manejo agrícola e nas ações para a conservação dos polinizadores nativos. Por esta razão, o serviço ambiental prestado pelos polinizadores foi considerado uma das prioridades de estudo para o século XXI e em 2000 foi 
criado um programa que visa a conservação e o uso sustentado de polinizadores (ver LAROCA \& ORTH, 2002), conhecido como "Iniciativa Internacional dos Polinizadores". No Brasil a "Iniciativa Brasileira de Polinizadores" (IBP) foi legalmente instituída em 2005 com o objetivo de: (i) planejar e definir ações para a conscientização da importância da polinização, (ii) fornecer subsídios para a formulação de políticas públicas que favoreçam o manejo, conservação e uso sustentável de polinizadores na agricultura, (iii) disseminar os conhecimentos gerados através de livros e websites, (iv) incrementar a capacitação de pessoas envolvidas com o tema e (v) avançar nos conhecimentos sobre polinização e polinizadores (IMPERATRIZ-FONSECA et al., 2007).

Tomando como foco a conservação de polinizadores, um dos resultados da IBP foi a publicação da Bibliografia Brasileira de Polinização e Polinizadores (MMA, 2006), levantamento detalhado da produção científica nacional sobre o tema. De acordo com este trabalho, o Pantanal aparece como o bioma com o menor número de estudos realizados. Considerando que o levantamento de espécies é o primeiro e essencial passo para avaliação do status de conservação, a falta dessa informação básica pode resultar em estratégias de conservação equivocadas, aumentando o risco de eventos irreversíveis de extinção de espécies.

Apresentamos a primeira listagem de visitantes florais para Mato Grosso do Sul (MS) com base na compilação de informações obtidas em estudos realizados no estado. Discutimos as principais lacunas e dificuldades existentes em termos de amostragem, o que certamente contribuirá para indicar áreas prioritárias para futuros estudos sobre o grupo, com consequente aumento do número de espécies registradas.

\section{MATERIAL E MÉTODOS}

O check list da entomofauna antófila foi elaborado a partir do levantamento bibliográfico sobre visitantes florais em periódicos científicos, boletins técnicos, livros, monografias de conclusão de curso, dissertações e teses, dentre outros. Foram considerados tanto trabalhos de levantamento como estudos de polinização. Os táxons não identificados até o nível específico foram inseridos à lista somente quando adicionavam nova família, tribo ou gênero. Os insetos foram organizados nos seguintes grupos: abelhas, besouros, borboletas/mariposas, formigas, hemípteros, moscas/mosquitos, vespas e outros. Os biomas foram classificados segundo IBGE (2004).

\section{RESULTADOS E DISCUSSÃO}

Lista das publicações e espécies para MS com comentários. Para o estado de MS foram registrados somente 17 estudos que trazem informações sobre visitantes florais, sendo sete artigos de periódicos, sete monografias/ dissertações e três capítulos de livros. A maioria dos estudos (10) gerou dados para apenas um grupo de insetos, geralmente abelhas (CONSOLARO, 2002; RoDRIGUES, 2002; ODA, 2003; VIEIRA et al., 2008; OliveIRA et al., 2007; MAIER, 2009;
FERreira et al., 2010; Longo \& Fischer, 2006; VILAs BoAS, 2009; AoKI et al., 2012). Outros incluíram também vespas (Boff, 2008; Auko et al., 2012), besouros e/ou esfingídeos (Munin et al., 2008). Quatro estudos contemplaram grupos de animais que não são considerados "polinizadores habituais" (AOKi \& Sigrist, 2006; Oliveira \& Sigrist, 2008; FaVA et al., 2011; SouzA, 2012), sendo que apenas um investiga a fauna antófila como um todo (AoKI \& Sigrist, 2006) (Tab. I).

A maioria dos estudos foi realizado no Cerrado (n $=11)$ e menos frequentemente no Pantanal $(n=6)$ (Fig. 1). Não foram encontrados estudos em áreas de com vegetação de Mata Atlântica, bioma restrito à região sul do estado. Dentre os estudos em Cerrado, nove foram realizados em remanescentes florestais inseridos em área urbana no centro (CONSOlaro, 2002; Rodrigues, 2002; OdA, 2003; Oliveira et al., 2007; MUNIN et al., 2008; OliveIRA \& SIGRIST, 2008; MAIER, 2009; Vilas BoAs, 2009) ou mais ao sul do estado (FERREIRA et al., 2010), com somente dois trabalhos realizados no nordeste de MS (AOKI \& SIGRIST, 2006; VieIRA et al., 2008) (Fig. 1). No Pantanal, há estudos na planície (LoNGo \& Fischer, 2006; BoFF, 2008; FAVA et al., 2011), incluindo um em formação chaquenha (SouZA, 2012) e, mais raramente, no Planalto (AoKI et al., 2012; Auko et al., 2012).

A compilação de dados revelou a ocorrência de dez grupos de insetos visitantes de flores, pertencentes a seis ordens, 80 famílias e que totalizaram 411 espécies (Tab. I). Contudo, ao menos 262 espécies que ocorrem no estado (Fig. 2), e permanecem como indeterminadas por conta de dificuldades taxonômicas, o que elevaria a riqueza para 673 espécies. Vespas e moscas estão entre os taxa com maior déficit na identificação. Maior riqueza foi amostrada para o Cerrado (307 spp.) que Pantanal (147 spp.), o que é razoável considerando o maior esforço amostral neste bioma, sendo registradas somente 43 espécies $(10,4 \%)$ em comum entre os dois biomas.

Abelhas foi o grupo mais rico (155 spp.), seguido por besouros (82), borboletas/mariposas (53) e vespas (45), que junto totalizaram $81,5 \%$ das espécies (Fig. 3). Maior riqueza de abelhas está relacionada ao fato da maioria dos trabalhos enfocar este grupo, além da especialização deste grupo na utilização de recursos florais. Abelhas possuem cuidado parental e o néctar como principal fonte de carboidratos e o pólen de proteína para a nutrição da prole e adultos (FAEGRI \& VAN DER PIJL, 1979; BARBOla et al., 2000). Surpreendente foi a segunda posição ocupada por besouros em relação a outros visitantes mais "ativos", como lepidópteros e moscas.

Dentre os visitantes categorizados como "polinizadores não habituais", destacaram-se hemípteros e formigas. Muitas espécies de percevejos são visitantes comuns e conspícuos, atraídos principalmente por néctar e tecidos florais, chegando muitas vezes a danificar o ovário (FAEGRI \& VAN DER PIJL, 1979). Por outro lado, formigas são comumente observadas em flores com néctar acessível e, raramente, são mencionadas sequer como visitantes florais, pois há o pressuposto que são polinizadores ineficientes ou, mais 
Tab. I. Insetos visitantes de flores amostrados em vegetação de Cerrado e Pantanal, no Mato Grosso do Sul, Brasil e referência bibliográfica: 1, Consolaro (2002); 2, Rodrigues (2002); 3, Oda (2003); 4, AoKi \& Sigrist (2006); 5, Oliveira et al. (2007); 6, OliveIRA \& SigRist (2008); 7, Munin et al. (2008); 8, Vieira et al. (2008); 9, Maier (2009); 10, Vilas Boas (2009); 11, Ferreira et al. (2010); 12, Longo \& Fischer (2006); 13, Boff (2008); 14, FaVa et al. (2011); 15, AoKi et al. (2012); 16, Auko et al. (2012); 17, Souza (2012).

\begin{tabular}{|c|c|c|c|}
\hline & Cerrado & Pantanal & Referências \\
\hline \multicolumn{4}{|l|}{ HYMENOPTERA, APOIDEA } \\
\hline \multicolumn{4}{|l|}{ ANDRENIDAE } \\
\hline Anthrenoides sp. & & $\mathrm{X}$ & 13 \\
\hline Cephalurgus anomalus Moure \& Lucas de Oliveira, 1962 & $\mathrm{X}$ & & 4 \\
\hline Oxaea flavescens Klug, 1807 & $\mathrm{X}$ & & $1,4,8$ \\
\hline \multicolumn{4}{|l|}{ APIDAE } \\
\hline Alepidosceles imitatrix (Schrottky, 1909) & $\mathrm{X}$ & $\mathrm{X}$ & $4,13,17$ \\
\hline Apis mellifera Linaeus, 1758 (introduzida) & $\mathrm{X}$ & $\mathrm{X}$ & $1,3,4,6,8,9,10,12,13,15,17$ \\
\hline Arhysoceble dichroopoda Moure, 1948 & $\mathrm{X}$ & & 4 \\
\hline Arhyzoceble sp. & $\mathrm{X}$ & & 4 \\
\hline Bombus (Fervidobombus) morio (Swederus, 1787) & $\mathrm{X}$ & $\mathrm{X}$ & $3,4,8,15$ \\
\hline Bombus (Fervidobombus) pauloensis Friese, 1913 & $\mathrm{X}$ & $\mathrm{X}$ & 1,13 \\
\hline Centris (Centris) aenea Lepeletier, 1841 & & $\mathrm{X}$ & 13 \\
\hline Centris (Centris) flavifrons (Fabricius, 1775) & $\mathrm{X}$ & $\mathrm{X}$ & $4,9,13$ \\
\hline Centris (Centris) nitens Lepeletier, 1841 & $\mathrm{X}$ & & 8 \\
\hline Centris (Centris) spilopoda Moure, 1969 & & $\mathrm{X}$ & 13 \\
\hline Centris (Centris) varia (Erichson, 1848) & $\mathrm{X}$ & $\mathrm{X}$ & $3,4,8,9,10,17$ \\
\hline Centris (Hemisiella) tarsata Smith, 1874 & & $\mathrm{X}$ & 15,17 \\
\hline Centris (Hemisiella) vittata Lepeletier, 1841 & & $\mathrm{X}$ & 15 \\
\hline Centris (Heterocentris) analis (Fabricius, 1804) & $\mathrm{X}$ & $\mathrm{X}$ & $1,3,15$ \\
\hline Centris (Melacentris) collaris Lepeletier, 1841 & $\mathrm{X}$ & & 4,8 \\
\hline Centris (Melacentris) xanthocnemis (Perty, 1833) & & $\mathrm{X}$ & 13,15 \\
\hline Centris (Trachina) fuscata Lepeletier, 1841 & & $\mathrm{X}$ & 13,17 \\
\hline Centris (Trachina) similis (Fabricius, 1804) & $\mathrm{X}$ & & 4 \\
\hline Centris (Xanthemisia) lutea Friese, 1899 & & $\mathrm{X}$ & 13 \\
\hline Cephalotrigona capitata (Smith, 1854) & $\mathrm{X}$ & & 4,8 \\
\hline Ceratina (Calloceratina) chloris Fabricius, 1804 & & $\mathrm{X}$ & 13 \\
\hline Ceratina (Crewella) diligens Smith, 1879 & & $\mathrm{X}$ & 15 \\
\hline Ceratina (Crewella) gossypii Schrottky, 1907 & $\mathrm{X}$ & & 4 \\
\hline Ceratina (Crewella) maculifrons Smith, 1854 & $\mathrm{X}$ & & 4 \\
\hline Ceratina (Crewella) morrensis Strand, 1910 & $\mathrm{X}$ & $\mathrm{X}$ & $9,13,15,17$ \\
\hline Ceratinula sp. & $\mathrm{X}$ & & 4 \\
\hline Dasihalonia sp. & $\mathrm{X}$ & & 4 \\
\hline Diadasina sp. & $\mathrm{X}$ & & 1 \\
\hline Epicharis (Epicharis) bicolor Smith 1854 & $\mathrm{X}$ & & 5 \\
\hline Epicharis (Epicharana) flava Friese, 1900 & $\mathrm{X}$ & & $1,3,4,5,9,10$ \\
\hline Epicharis (Epicharana) rustica (Olivier, 1789) & $\mathrm{X}$ & & 8 \\
\hline Epicharis (Epicharitides) cockerelli Friese, 1900 & $\mathrm{X}$ & & 5,9 \\
\hline Epicharis (Epicharoides) xanthogastra Moure \& Seabra, 1959 & $\mathrm{X}$ & & 3 \\
\hline Epicharis (Hoplepicharis) affinis Smith, 1874 & $\mathrm{X}$ & & 4,5 \\
\hline Epicharis (Triepicharis) analis Lepeletier, 1841 & $\mathrm{X}$ & & 4,5 \\
\hline Eufriesea sp. & $\mathrm{X}$ & & 1 \\
\hline Euglossa (Euglossa) melanotricha Moure, 1967 & $\mathrm{X}$ & & 4 \\
\hline Euglossa (Euglossa) townsendi Cockerell, 1904 & $\mathrm{X}$ & & 9 \\
\hline Euglossa (Euglossella) viridis (Perty, 1833) & $\mathrm{X}$ & & 4 \\
\hline Eulaema (Apeulaema) nigrita Lepeletier, 1841 & $\mathrm{X}$ & $\mathrm{X}$ & $1,2,15$ \\
\hline Exaerete smaragdina (Guérin, 1844) & $X$ & & 1 \\
\hline Exomalopsis (Exomalopsis) analis Spinola, 1853 & & $\mathrm{X}$ & 13 \\
\hline Exomalopsis (Exomalopsis) auropilosa Spinola, 1853 & $\mathrm{X}$ & & 1,8 \\
\hline Exomalopsis (Exomalopsis) fulvofasciata Smith, 1879 & $\mathrm{X}$ & & 4,8 \\
\hline Frieseomelitta trichocerata Moure, 1990 & $\mathrm{X}$ & & 4 \\
\hline Frieseomelitta varia (Lepeletier, 1836) & $\mathrm{X}$ & & 1,8 \\
\hline Geotrigona mombuca (Smith, 1863) & $\mathrm{X}$ & & 9 \\
\hline Hopliphora velutina (Lepeletier \& Serville, 1825) & & $\mathrm{X}$ & 15 \\
\hline Leurotrigona muelleri (Friese, 1900) & $\mathrm{X}$ & & 3,9 \\
\hline
\end{tabular}


Tab. I. Cont.

\begin{tabular}{|c|c|c|c|}
\hline & Cerrado & Pantanal & Referências \\
\hline Lophopedia sp. & $\mathrm{X}$ & & 3 \\
\hline Melipona (Melipona) subnitida Ducke, 1910 & $\mathrm{X}$ & & 3 \\
\hline Melipona (Michmelia) rufiventris Lepeletier, 1836 & $\mathrm{X}$ & $\mathrm{X}$ & 4,15 \\
\hline Melipona orbignyi (Guérin, 1844) & & $\mathrm{X}$ & 15 \\
\hline Melissodes (Ecplectica) sexcincta (Lepeletier, 1841) & $\mathrm{X}$ & & 4 \\
\hline Melissoptila cnecomala (Moure, 1944) & $\mathrm{X}$ & & 8 \\
\hline Melissoptila richardiae Bertoni \& Schrottky, 1910 & $\mathrm{X}$ & & 4 \\
\hline Melitoma segmentaria (Fabricius, 1804) & $\mathrm{X}$ & & 1 \\
\hline Monoeca lanei (Moure, 1944) & $\mathrm{X}$ & & 4 \\
\hline Nannotrigona testaceicornis (Lepeletier, 1836) & $\mathrm{X}$ & $\mathrm{X}$ & 8,13 \\
\hline Nomada sp. & $\mathrm{X}$ & & 4,9 \\
\hline Osirinus santiagoi (Almeida, 1996) & $\mathrm{X}$ & & 4 \\
\hline Oxytrigona sp. & $\mathrm{X}$ & & 8 \\
\hline Paratetrapedia fervida (Smith, 1879) & $\mathrm{X}$ & & 1 \\
\hline Paratetrapedia gigantea (Schrottky, 1909) & $\mathrm{X}$ & & 4 \\
\hline Paratetrapedia maculata Friese, 1899 & $\mathrm{X}$ & & 4 \\
\hline Paratrigona lineata (Lepeletier, 1836) & $\mathrm{X}$ & $\mathrm{X}$ & $3,4,5,6,8,9,10,13$ \\
\hline Partamona sp. & $\mathrm{X}$ & & 3,4 \\
\hline Plebeia catamarcensis (Holmberg, 1903) & & $\mathrm{X}$ & 15 \\
\hline Plebeia droryana (Friese, 1900) & $\mathrm{X}$ & & 9 \\
\hline Plebeia remota (Holmberg, 1903) & $\mathrm{X}$ & & 9 \\
\hline Rhathymus sp. & $\mathrm{X}$ & & 4 \\
\hline Scaptotrigona bipunctata (Lepeletier, 1836) & $\mathrm{X}$ & & 3 \\
\hline Scaptotrigona depilis (Moure, 1942) & $\mathrm{X}$ & & 11 \\
\hline Scaptotrigona jujuyensis (Schrottky, 1911) & & $\mathrm{X}$ & 15 \\
\hline Scaptotrigona postica (Latreille, 1807) & $\mathrm{X}$ & $\mathrm{X}$ & $4,9,15$ \\
\hline Scaura latitarsis (Friese, 1900) & $\mathrm{X}$ & $\mathrm{X}$ & 4,15 \\
\hline Scaura longula (Lepeletier, 1836) & $\mathrm{X}$ & & 4 \\
\hline Scaura timida (Silvestri, 1902) & & $\mathrm{X}$ & 15 \\
\hline Schwarziana quadripunctata (Lepeletier, 1836) & $\mathrm{X}$ & & 4 \\
\hline Tetragona clavipes (Fabricius, 1804) & $\mathrm{X}$ & & 4 \\
\hline Tetragonisca angustula (Latreille, 1811) & $\mathrm{X}$ & $\mathrm{X}$ & $1,3,4,8,9,10,13,15$ \\
\hline Tetragonisca fiebrigi (Schwarz, 1938) & & $\mathrm{X}$ & 17 \\
\hline Tetrapedia aff. amplitarsis Friese, 1899 & $\mathrm{X}$ & & 3 \\
\hline Tetrapedia aff. curvipes Friese, 1990 & $\mathrm{X}$ & & 3 \\
\hline Tetrapedia diversipes Klug, 1810 & $\mathrm{X}$ & & 5 \\
\hline Tetrapedia hypoleuca Moure, 1999 & $\mathrm{X}$ & & 4 \\
\hline Tetrapedia imitatrix Moure, 1999 & $\mathrm{X}$ & $\mathrm{X}$ & $4,9,10,15$ \\
\hline Tetrapedia plumipes Smith, 1879 & $\mathrm{X}$ & & 4 \\
\hline Tetrapedia rugulosa Friese, 1899 & $\mathrm{X}$ & & 8 \\
\hline Thalestria spinosa (Fabricius, 1804) & $\mathrm{X}$ & & 4 \\
\hline Thygater (Thygater) analis (Lepeletier, 1841) & & $\mathrm{X}$ & 15 \\
\hline Trigona amalthea (Olivier, 1789) & $\mathrm{X}$ & & 8 \\
\hline Trigona amazonensis (Ducke, 1916) & $\mathrm{X}$ & & 4 \\
\hline Trigona branneri Cockerell, 1912 & $\mathrm{X}$ & & $4,5,6$ \\
\hline Trigona chanchamayoensis Schwarz, 1948 & & $\mathrm{X}$ & 15 \\
\hline Trigona fulviventris Guérin, 1835 & $\mathrm{X}$ & $\mathrm{X}$ & 4,15 \\
\hline Trigona fuscipennis Friese, 1900 & $\mathrm{X}$ & $\mathrm{X}$ & $4,13,15$ \\
\hline Trigona hyalinata (Lepeletier, 1836) & $\mathrm{X}$ & & 1,8 \\
\hline Trigona recursa Smith, 1863 & $\mathrm{X}$ & $\mathrm{X}$ & $3,9,15$ \\
\hline Trigona spinipes (Fabricius, 1793) & $\mathrm{X}$ & $\mathrm{X}$ & $1,3,5,8,9,10,12,13,14$ \\
\hline Xanthopedia affinis Larocca \& Moure & $\mathrm{X}$ & & 4 \\
\hline Xanthopedia iheringii (Friese, 1899) & $\mathrm{X}$ & & 4,9 \\
\hline Xanthopedia larocai Moure, 1992 & $\mathrm{X}$ & & $4,5,9,10$ \\
\hline Xylocopa (Neoxylocopa) frontalis (Olivier, 1789) & $\mathrm{X}$ & $\mathrm{X}$ & $1,13,15$ \\
\hline Xylocopa (Neoxylocopa) grisescens Lepeletier, 1841 & $\mathrm{X}$ & & 8 \\
\hline Xylocopa (Neoxylocopa) ordinaria Smith, 1874 & $\mathrm{X}$ & $\mathrm{X}$ & $9,10,13$ \\
\hline Xylocopa (Neoxylocopa) suspecta Moure \& Camargo, 1988 & $\mathrm{X}$ & $\mathrm{X}$ & $3,4,6,17$ \\
\hline
\end{tabular}


Tab. I. Cont.

\begin{tabular}{|c|c|c|c|}
\hline & Cerrado & Pantanal & Referências \\
\hline Xylocopa (Schonnherria) muscaria (Fabricius, 1775) & & $\mathrm{X}$ & 15 \\
\hline Xylocopa (Schonnherria) splendidula Lepeletier, 1841 & & $\mathrm{X}$ & 17 \\
\hline \multicolumn{4}{|l|}{ COLLETIDAE } \\
\hline Colletes sp. & $\mathrm{X}$ & & 4,9 \\
\hline Hylaeus sp. & $\mathrm{X}$ & & 1 \\
\hline Ptiloglossa willinki (Moure, 1953) & & $\mathrm{X}$ & 17 \\
\hline Tetraglossula bigamica (Strand, 1910) & $\mathrm{X}$ & & 1,4 \\
\hline \multicolumn{4}{|l|}{ HALICTIDAE } \\
\hline Agapostemon chapadensis Cockerell, 1900 & $\mathrm{X}$ & & 4 \\
\hline Augochlora amphitrite (Schrottky, 1909) & $\mathrm{X}$ & & 4 \\
\hline Augochlora foxiana Cockerell, 1900 & $\mathrm{X}$ & & 8 \\
\hline Augochlora mulleri Cockerell, 1900 & $\mathrm{X}$ & $\mathrm{X}$ & 4,15 \\
\hline Augochlora semiramis (Schrottky, 1910) & $\mathrm{X}$ & & 9 \\
\hline Augochlorella iopoecila Moure, 1950 & $\mathrm{X}$ & & 4 \\
\hline Augochloropsis aff. multiplex (Vachal, 1903) & $\mathrm{X}$ & & 9 \\
\hline Augochloropsis callichroa (Cockerell, 1900) & $\mathrm{X}$ & & $4,6,9$ \\
\hline Augochloropsis cleopatra (Schrottky, 1902) & $\mathrm{X}$ & & 4 \\
\hline Augochloropsis cupreola (Cockerell, 1900) & $\mathrm{X}$ & & 8 \\
\hline Augochloropsis deianira Schrottky, 1910 & & $\mathrm{X}$ & 13 \\
\hline Augochloropsis semiramis (Jörgensen, 1912) & $\mathrm{X}$ & & 4 \\
\hline Augochloropsis smithiana (Cockerell, 1900) & $\mathrm{X}$ & & 9 \\
\hline Augochloropsis sparsilis (Vachal, 1903) & & $\mathrm{X}$ & 15 \\
\hline Augochloropsis wallacei (Cockerell, 1900) & $\mathrm{X}$ & & 4 \\
\hline Dialictus osmioides (Ducke, 1902) & $\mathrm{X}$ & & 4 \\
\hline Dialictus phaedrus (Schrottky, 1910) & $\mathrm{X}$ & & 4 \\
\hline Dialictus ypirangensis (Schrottky, 1910) & & $\mathrm{X}$ & 13 \\
\hline Neocorynura aff. aenigma (Gribodo, 1894) & $\mathrm{X}$ & & 4 \\
\hline Paraxystoglossa jocasta (Schrottky,1910) & $\mathrm{X}$ & & 4 \\
\hline Pseudagapostemon (Pseudagapostemon) pissisi (Vachal, 1903) & $\mathrm{X}$ & & 4 \\
\hline Pseudaugochlora graminea (Fabricius, 1804) & $\mathrm{X}$ & & $4,6,8$ \\
\hline Rhectomia pumilla Moure, 1947 & $\mathrm{X}$ & & 4 \\
\hline Temnosoma sp. & $\mathrm{X}$ & & 4 \\
\hline Thectochlora alaris (Vachal, 1904) & $\mathrm{X}$ & & 4 \\
\hline \multicolumn{4}{|l|}{ MEGACHILIDAE } \\
\hline Anthodioctes megachiloides Holmberg, 1903 & $\mathrm{X}$ & & 4 \\
\hline Coelioxys (Acrocoelioxys) otomita Cresson, 1878 & & $\mathrm{X}$ & 13,15 \\
\hline Coelioxys (Acrocoelioxys) tolteca Cresson, 1878 & & $\mathrm{X}$ & 13 \\
\hline Coelioxys (Glyptocoelioxys) pampeana Holmberg, 1887 & & $\mathrm{X}$ & 13 \\
\hline Coelioxys (Neocoelioxys) praetextata Haliday, 1836 & $\mathrm{X}$ & & 4 \\
\hline Coelioxys vidua Smith, 1854 & & $\mathrm{X}$ & 13,15 \\
\hline Epanthidium tigrinum (Schrottky, 1905) & $\mathrm{X}$ & $\mathrm{X}$ & 1,15 \\
\hline Hypanthidioides aff. gregaria (Schrottky, 1905) & & $\mathrm{X}$ & 15 \\
\hline Hypanthidium sp. & $\mathrm{X}$ & & 4,8 \\
\hline Larocanthidium nigritulum Urban, 1997 & $\mathrm{X}$ & $\mathrm{X}$ & $4,13,15$ \\
\hline Megachile (Leptorachis) aetheria Mitchell, 1930 & & $\mathrm{X}$ & 13 \\
\hline Megachile (Tylomegachile) orba Schrottky, 1913 & & $\mathrm{X}$ & 13 \\
\hline Megachile aff. paulistana Schrottky, 1902 & $\mathrm{X}$ & $\mathrm{X}$ & 4,13 \\
\hline Megachile lentifera Vachal, 1909 & & $\mathrm{X}$ & 15 \\
\hline \multicolumn{4}{|l|}{ COLEOPTERA } \\
\hline \multicolumn{4}{|l|}{ ANTHICIDAE } \\
\hline Eurygeniinae sp. & $\mathrm{X}$ & & 4 \\
\hline \multicolumn{4}{|l|}{ BRENTIDAE } \\
\hline Apion sp. & $\mathrm{X}$ & & 4 \\
\hline \multicolumn{4}{|l|}{ BUPRESTIDAE } \\
\hline Agrilus sp. & $\mathrm{X}$ & $\mathrm{X}$ & 4,17 \\
\hline Tetragonoschema missionarium Obenberger, 1947 & & $\mathrm{X}$ & 17 \\
\hline \multicolumn{4}{|l|}{ CANTHARIDAE } \\
\hline Cantharis sp. & $\mathrm{X}$ & & 4 \\
\hline
\end{tabular}


Tab. I. Cont.

\begin{tabular}{|c|c|c|c|}
\hline & Cerrado & Pantanal & Referências \\
\hline Chauliognathus fallax Germar, 1824 & $\mathrm{X}$ & & 4 \\
\hline \multicolumn{4}{|l|}{ CERAMBYCIDAE } \\
\hline Chrysoprasis aurigena Germar, 1824 & $\mathrm{X}$ & & 4 \\
\hline Chrysoprasis chalybea Redtenbacher, 1868 & $\mathrm{X}$ & & 4 \\
\hline Chrysoprasis hypocrita Erichson,1847 & $\mathrm{X}$ & & 4 \\
\hline Cosmisoma cyaneum cyaneum Gounelle, 1891 & $\mathrm{X}$ & & 4 \\
\hline Mallosoma zonata Sahlberg, 1823 & $\mathrm{X}$ & & 4 \\
\hline Paratenthras martinsi Monné, 1998 & & $\mathrm{X}$ & 14 \\
\hline \multicolumn{4}{|l|}{ CHRYSOMELIDAE } \\
\hline Acanthoscelides sp. & $\mathrm{X}$ & & 4 \\
\hline Alticini sp. & $\mathrm{X}$ & & 4 \\
\hline Attalus sp. & & $\mathrm{X}$ & 17 \\
\hline Babiohaltica spp. & & $\mathrm{X}$ & 17 \\
\hline Bruchini sp. & & $\mathrm{X}$ & 17 \\
\hline Chrysodina spp. & $\mathrm{X}$ & & 4 \\
\hline Colaspis sp. & $\mathrm{X}$ & & 4 \\
\hline Diabrotica sp. & $\mathrm{X}$ & & 4 \\
\hline Diabrotica speciosa Germar, 1824 & $\mathrm{X}$ & & 4 \\
\hline Diabroticini sp. & $\mathrm{X}$ & & 4 \\
\hline Eumolpinae sp. & $\mathrm{X}$ & & 4 \\
\hline Iphimeis sp. & $\mathrm{X}$ & & 4 \\
\hline Lema sp. & & $\mathrm{X}$ & 17 \\
\hline Lilophaea sp. & $\mathrm{X}$ & & 4 \\
\hline Metallactus sp. & & $\mathrm{X}$ & 17 \\
\hline Monachini sp. & $\mathrm{X}$ & & 4 \\
\hline Omophoita sp. & $\mathrm{X}$ & & 4 \\
\hline Pachybrachini sp. & $\mathrm{X}$ & & 4 \\
\hline Spintherophyta sp. & & $\mathrm{X}$ & 17 \\
\hline Urodera circumcincta Lacordaire, 1848 & $\mathrm{X}$ & & 4 \\
\hline \multicolumn{4}{|l|}{ COCCINELLIDAE } \\
\hline Hyperaspis sp. & $\mathrm{X}$ & & 4 \\
\hline \multicolumn{4}{|l|}{ CURCULIONIDAE } \\
\hline Anthonomus sp. & $\mathrm{X}$ & & 4 \\
\hline Atrichis sp. & $\mathrm{X}$ & & 4 \\
\hline Baridinae sp. & $\mathrm{X}$ & & 4 \\
\hline Belopoeus spp. & & $\mathrm{X}$ & 14 \\
\hline Celetes sp. & & $\mathrm{X}$ & 14 \\
\hline Conotrachelus sp. & $\mathrm{X}$ & & 4 \\
\hline Derelomus sp. & & $\mathrm{X}$ & 14 \\
\hline Erirrhinini sp. & $\mathrm{X}$ & & 4 \\
\hline Erodiscus proximus Viana, 1959 & $\mathrm{X}$ & & 4 \\
\hline Eudiagogini spp. & & $\mathrm{X}$ & 17 \\
\hline Geraeus sp. & & $\mathrm{X}$ & 17 \\
\hline Hylobiini sp. & & $\mathrm{X}$ & 14 \\
\hline Hypothenemus sp. & & $\mathrm{X}$ & 14 \\
\hline Madarini spp. & & $\mathrm{X}$ & 14 \\
\hline Naupactus sp. & $\mathrm{X}$ & & 4 \\
\hline Parapantomorus fluctuosus Boheman, 1840 & $\mathrm{X}$ & & 4 \\
\hline Phyrdenus sp. & $\mathrm{X}$ & & 4 \\
\hline Prionomerus sp. & $\mathrm{X}$ & & 4 \\
\hline \multicolumn{4}{|l|}{ DERMESTIDAE } \\
\hline Cryptorhopalum sp. & $\mathrm{X}$ & & 4 \\
\hline \multicolumn{4}{|l|}{ MELANDRYIDAE } \\
\hline Epitragus sp. & & $\mathrm{X}$ & 17 \\
\hline \multicolumn{4}{|l|}{ MELYRIDAE } \\
\hline Astylus variegatus Germar, 1824 & $\mathrm{X}$ & & 4 \\
\hline \multicolumn{4}{|l|}{ MORDELLIDAE } \\
\hline Mordella sp. & & $\mathrm{X}$ & 17 \\
\hline
\end{tabular}


Tab. I. Cont.

\begin{tabular}{|c|c|c|c|}
\hline & Cerrado & Pantanal & Referências \\
\hline \multicolumn{4}{|l|}{ NITIDULIDAE } \\
\hline Camptodes vittata Erichson, 1843 & & $\mathrm{X}$ & 17 \\
\hline Carpophilus sp. & $\mathrm{X}$ & & 4 \\
\hline Colopterus sp. & $\mathrm{X}$ & $\mathrm{X}$ & 4,14 \\
\hline Conotelus sp. & $\mathrm{X}$ & & 4 \\
\hline Lobiopa sp. & $\mathrm{X}$ & & 4 \\
\hline Mystrops sp. & & $\mathrm{X}$ & 14 \\
\hline \multicolumn{4}{|l|}{ RHIPIPHORIDAE } \\
\hline Macrosiagon sp. & $\mathrm{X}$ & & 4 \\
\hline \multicolumn{4}{|l|}{ SCARABAEIDAE } \\
\hline Cyclocephala quatuordecimpunctata Mannhenheim, 1829 & $\mathrm{X}$ & & 4 \\
\hline Cyclocephala paraguayensis Arrow, 1904 & $\mathrm{X}$ & & 7 \\
\hline Cyclocephala pugnax Arrow, 1914 & & $\mathrm{X}$ & 14 \\
\hline Cyclocephala sp. & $\mathrm{X}$ & & 4 \\
\hline Dicrania sp. & $\mathrm{X}$ & & 4 \\
\hline Macraspis festiva Burmeister, 1844 & $\mathrm{X}$ & & 4 \\
\hline Macraspis morio Burmeister, 1844 & $\mathrm{X}$ & & 4 \\
\hline Macraspis sp. & $\mathrm{X}$ & & 4 \\
\hline Macraspis thoracica Mannhenheim, 1829 & $\mathrm{X}$ & & 4 \\
\hline Macrodactylus pumillo Burmeister, 1855 & $\mathrm{X}$ & & 4 \\
\hline Symmela sp. & $\mathrm{X}$ & & 4 \\
\hline \multicolumn{4}{|l|}{ STAPHYLINIDAE } \\
\hline Atheta sp. & & $\mathrm{X}$ & 14 \\
\hline Eulissus chalybaeus Mannerheim, 1830 & $\mathrm{X}$ & & 4 \\
\hline \multicolumn{4}{|l|}{ TENEBRIONIDAE } \\
\hline Alleculinae sp. & $\mathrm{X}$ & & 4 \\
\hline Lagria villosa Fabricius, 1781 (introduzida) & $\mathrm{X}$ & & 4 \\
\hline Lagriini sp. & $\mathrm{X}$ & & 4 \\
\hline Lystronychus sp. & $\mathrm{X}$ & & 4 \\
\hline Paratenetus sp. & & $\mathrm{X}$ & 14 \\
\hline Strongylium sp. & $\mathrm{X}$ & & 4 \\
\hline \multicolumn{4}{|l|}{ TROGOSSITIDAE } \\
\hline Tenebroides sp. & & $\mathrm{X}$ & 14 \\
\hline \multicolumn{4}{|l|}{ LEPIDOPTERA } \\
\hline \multicolumn{4}{|l|}{ HESPERIIDAE } \\
\hline Aides epitus Stoll 1781 & $\mathrm{X}$ & & 2 \\
\hline Antigonus sp. & $\mathrm{X}$ & & 4 \\
\hline Anisochoria sp. & $\mathrm{X}$ & & 4 \\
\hline Chioides catillus (Cramer, 1779) & & $\mathrm{X}$ & 17 \\
\hline Heliopetes arsalte Linnaeus, 1758 & $\mathrm{X}$ & & 4 \\
\hline Heliopetes macaira Reakirt, 1866 & $\mathrm{X}$ & & 4 \\
\hline Heliopetes omrina Butler, 1870 & $\mathrm{X}$ & & 4 \\
\hline Mysoria barcastus Sepp, 1855 & $\mathrm{X}$ & & 4 \\
\hline Panoquina sp. & $\mathrm{X}$ & & 4 \\
\hline Polites vibex Geyer, 1832 & $\mathrm{X}$ & & 4 \\
\hline Pompeius pompeius Latreille, 1824 & $\mathrm{X}$ & & 4 \\
\hline Proteides mercurius Fabricius, 1787 & $\mathrm{X}$ & & 4 \\
\hline Pyrgus oileus Linnaeus, 1767 & $\mathrm{X}$ & & 4 \\
\hline Typhedanus undulatus Hewitson, 1867 & $\mathrm{X}$ & & 4 \\
\hline Urbanus dorantes (Stoll, 1790) & & $\mathrm{X}$ & 17 \\
\hline Vehilius stictomenes Butler, 1877 & $\mathrm{X}$ & & 4 \\
\hline \multicolumn{4}{|l|}{ LYCAENIDAE } \\
\hline Audre sp. & $\mathrm{X}$ & & 4 \\
\hline Chlorostrymon sp. & $\mathrm{X}$ & & 4 \\
\hline Ematurgina sp. & $\mathrm{X}$ & & 4 \\
\hline Hemiargus hanno Stoll, 1780 & $\mathrm{X}$ & $\mathrm{X}$ & 4,17 \\
\hline cf. Janthecla $\mathrm{sp}$. & $\mathrm{X}$ & & 4 \\
\hline Leptotes cassius Cramer, 1775 & $\mathrm{X}$ & & 4 \\
\hline
\end{tabular}


Tab. I. Cont.

\begin{tabular}{|c|c|c|c|}
\hline & Cerrado & Pantanal & Referências \\
\hline Rekoa palegon Stoll, 1780 & $\mathrm{X}$ & & 4 \\
\hline Stalactis phlegia Cramer, 1765 & $\mathrm{X}$ & & 4 \\
\hline Strymon sp. & $\mathrm{X}$ & $\mathrm{X}$ & 4,17 \\
\hline Eumaeinae & $\mathrm{X}$ & & 4 \\
\hline \multicolumn{4}{|l|}{ cf. MIMMALONIDAE } \\
\hline cf. Mimmalonidae & $\mathrm{X}$ & & 7 \\
\hline \multicolumn{4}{|l|}{ NYMPHALIDAE } \\
\hline Actinote sp. & $\mathrm{X}$ & & 4 \\
\hline Agraulis vanillae maculosa (Stichel, [1908]) & & $\mathrm{X}$ & 17 \\
\hline Anartia jatrophae Johansson, 1763 & $\mathrm{X}$ & & 4 \\
\hline Anthanassa frisia herma (W.C. Hewitson, 1864) & & $\mathrm{X}$ & 17 \\
\hline Chlosyne lacinia Geyer, 1837 & $\mathrm{X}$ & & 4 \\
\hline Danaus gilippus Cramer, 1776 & $\mathrm{X}$ & & 4 \\
\hline Dryadula phaetusa Linnaeus, 1758 & $\mathrm{X}$ & & 4 \\
\hline Eunica ingens Seitz, 1915 & $\mathrm{X}$ & & 4 \\
\hline Euptoieta hegesia Cramer, 1780 & $\mathrm{X}$ & & 4 \\
\hline Hermeuptychia hermes Fabricius, 1775 & $\mathrm{X}$ & & 4 \\
\hline Junonia genoveva Stoll, 1782 & $\mathrm{X}$ & & 4 \\
\hline Marpesia chiron Fabricius, 1775 & $\mathrm{X}$ & & 4 \\
\hline Phystis simois variegata (J.K.M. Röber, 1913) & & $\mathrm{X}$ & 17 \\
\hline Tegosa sp. & $\mathrm{X}$ & & 4 \\
\hline Yphthimoides affinis Butler, 1867 & $\mathrm{X}$ & & 4 \\
\hline Yphthimoides sp. & $\mathrm{X}$ & & 4 \\
\hline \multicolumn{4}{|l|}{ PIERIDAE } \\
\hline Ascia monuste (Linnaeus, 1764) & & $\mathrm{X}$ & 17 \\
\hline Eurema albula Cramer, 1775 & $\mathrm{X}$ & & 4 \\
\hline Eurema elathea Cramer, 1777 & $\mathrm{X}$ & $\mathrm{X}$ & 4,17 \\
\hline Glutophrissa drusilla drusilla (Cramer 1777) & & $\mathrm{X}$ & 17 \\
\hline Phoebis sennae Linnaeus, 1758 & $\mathrm{X}$ & & 2,4 \\
\hline Phoebis statira Cramer, 1777 & $\mathrm{X}$ & & 4 \\
\hline Pyrisitia leuce leuce (Boisduval, 1836) & & $\mathrm{X}$ & 17 \\
\hline \multicolumn{4}{|l|}{ RIODINIDAE } \\
\hline Apodemia sp. & & $\mathrm{X}$ & 17 \\
\hline Aricoris sp. & & $\mathrm{X}$ & 17 \\
\hline \multicolumn{4}{|l|}{ SPHINGIDAE } \\
\hline Agrius cingulatus (Fabricius, 1775) & $\mathrm{X}$ & & 7 \\
\hline \multicolumn{4}{|l|}{ HYMENOPTERA, FORMICIDAE } \\
\hline \multicolumn{4}{|l|}{ Dolichoderinae } \\
\hline Azteca sp. & $\mathrm{X}$ & & 4 \\
\hline Dolichoderus bispinosus (Olivier 1792) & & $\mathrm{X}$ & 17 \\
\hline Dorymyrmex thoracicus Gallardo, 1916 & & $\mathrm{X}$ & 17 \\
\hline Linepithema sp. & $\mathrm{X}$ & & 4 \\
\hline \multicolumn{4}{|l|}{ Ectatomminae } \\
\hline Ectatomma brunneum Smith, 1858 & $\mathrm{X}$ & & 4 \\
\hline Ectatomma tuberculatum Olivier, 1792 & $\mathrm{X}$ & & 4 \\
\hline Ectatomma spp. & $\mathrm{X}$ & & 4 \\
\hline \multicolumn{4}{|l|}{ Formicinae } \\
\hline Camponotus crassus Mayr, 1862 & & $\mathrm{X}$ & 17 \\
\hline Camponotus crispulus Wild, 2007 & & $\mathrm{X}$ & 17 \\
\hline Camponotus mus Roger, 1863 & & $\mathrm{X}$ & 17 \\
\hline Camponotus rufipes Fabricius, 1775 & $\mathrm{X}$ & & 4 \\
\hline Camponotus sericeiventris Guérin-Méneville, 1838 & $\mathrm{X}$ & & 4 \\
\hline Nylanderia fulva (Mayr, 1862) & & $\mathrm{X}$ & 17 \\
\hline \multicolumn{4}{|l|}{ Myrmicinae } \\
\hline Atta rubropilosa Forel, 1908 & $\mathrm{X}$ & & 4 \\
\hline Cephalotes atratus Linnaeus, 1758 & $\mathrm{X}$ & & 4 \\
\hline Cephalotes pusillus (Klug, 1824) & & $\mathrm{X}$ & 17 \\
\hline
\end{tabular}


Tab. I. Cont.

\begin{tabular}{|c|c|c|c|}
\hline & Cerrado & Pantanal & Referências \\
\hline Crematogaster spp. & $\mathrm{X}$ & & 4 \\
\hline Pheidole gertrudae Forel, 1886 & $\mathrm{X}$ & & 4 \\
\hline Solenopsis sp. & $\mathrm{X}$ & & 4 \\
\hline Wasmannia sp. & $\mathrm{X}$ & & 4 \\
\hline \multicolumn{4}{|l|}{ Ponerinae } \\
\hline Pachycondyla sp. & $\mathrm{X}$ & & 4 \\
\hline \multicolumn{4}{|l|}{ Pseudomyrmecinae } \\
\hline Pseudomyrmex gracilis (Fabricius, 1804) & & $\mathrm{X}$ & 17 \\
\hline Pseudomyrmex spp. & $\mathrm{X}$ & & 4 \\
\hline \multicolumn{4}{|l|}{ HEMÍPTERA } \\
\hline \multicolumn{4}{|l|}{ CERCOPIDAE } \\
\hline Cercopidae spp. & $\mathrm{X}$ & & 4 \\
\hline \multicolumn{4}{|l|}{ COREIDAE } \\
\hline Allocoris sp. & $\mathrm{X}$ & & 4 \\
\hline Coreidae spp. & $\mathrm{X}$ & & 4 \\
\hline \multicolumn{4}{|l|}{ FULGORIDAE } \\
\hline Fulgoridae spp. & $\mathrm{X}$ & & 4 \\
\hline \multicolumn{4}{|l|}{ LYGAEIDAE } \\
\hline Lygaeidae spp. & $\mathrm{X}$ & & 4 \\
\hline \multicolumn{4}{|l|}{ MEMBRACIDAE } \\
\hline Membracidae spp. & $\mathrm{X}$ & & 4 \\
\hline \multicolumn{4}{|l|}{ MIRIDAE } \\
\hline Miridae spp. & $\mathrm{X}$ & & 4 \\
\hline \multicolumn{4}{|l|}{ PENTATOMIDAE } \\
\hline Euschistus (Euschistus) heros (Fabricius, 1798) & $\mathrm{X}$ & & 4 \\
\hline Sibaria armata (Dallas, 1851) & $\mathrm{X}$ & & 4 \\
\hline Thyanta (Thyanta) perditor (Fabricius, 1794) & $\mathrm{X}$ & & 4 \\
\hline \multicolumn{4}{|l|}{ REDUVIIDAE } \\
\hline Reduviidae spp. & $\mathrm{X}$ & & 4 \\
\hline \multicolumn{4}{|l|}{ RHOPALIDAE } \\
\hline Rhopalidae & $\mathrm{X}$ & & 4 \\
\hline \multicolumn{4}{|l|}{ SCUTELLERIDAE } \\
\hline Orsilochides leucoptera (Germar, 1839) & $\mathrm{X}$ & & 4 \\
\hline Scutelleridae sp. & & $\mathrm{X}$ & 17 \\
\hline \multicolumn{4}{|l|}{ Sternorrhyncha } \\
\hline Sternorrhyncha spp. & $\mathrm{X}$ & & 4 \\
\hline \multicolumn{4}{|l|}{ DIPTERA } \\
\hline \multicolumn{4}{|l|}{ ASILIDAE } \\
\hline Asilidae sp. & & $\mathrm{X}$ & 17 \\
\hline \multicolumn{4}{|l|}{ BIBIONIDAE } \\
\hline Bibionidae sp. & $\mathrm{X}$ & & 4 \\
\hline \multicolumn{4}{|l|}{ BOMBYLIIDAE } \\
\hline Chrysanthrax spp. & $\mathrm{X}$ & $\mathrm{X}$ & 4,17 \\
\hline Exoprosopa sp. & $\mathrm{X}$ & $\mathrm{X}$ & 4,17 \\
\hline Poecilognathus spp. & $\mathrm{X}$ & $\mathrm{X}$ & 4,17 \\
\hline Toxophora aurea Macquart, 1848 & & $\mathrm{X}$ & 17 \\
\hline Villa sp. & $\mathrm{X}$ & & 4 \\
\hline CALLIPHORIDAE & & & \\
\hline Chloroprocta idioidea (Robineau-Desvoidy, 1830) & & $\mathrm{X}$ & 17 \\
\hline Chrysomya sp. & $\mathrm{X}$ & & 4 \\
\hline Hemilucilia segmentaria (Fabricius, 1805) & & $\mathrm{X}$ & 17 \\
\hline CAMILLIDAE & & & \\
\hline Camillidae sp. & $\mathrm{X}$ & & 4 \\
\hline CARNIDAE & & & \\
\hline Carnidae sp. & $\mathrm{X}$ & & 4 \\
\hline CHLOROPIDAE & & & \\
\hline Chloropidae spp. & & $\mathrm{X}$ & 17 \\
\hline
\end{tabular}


Tab. I. Cont.

\begin{tabular}{|c|c|c|c|}
\hline & Cerrado & Pantanal & Referências \\
\hline Olcella $\mathrm{sp}$. & & $\mathrm{X}$ & 17 \\
\hline \multicolumn{4}{|l|}{ CULICIDAE } \\
\hline Culicidae sp. & & $\mathrm{X}$ & 17 \\
\hline \multicolumn{4}{|l|}{ DOLICHOPODIDAE } \\
\hline Dolichopodidae spp. & $\mathrm{X}$ & & 4 \\
\hline \multicolumn{4}{|l|}{ DROSOPHILIDAE } \\
\hline Drosophila sp. & & $\mathrm{X}$ & 14 \\
\hline \multicolumn{4}{|l|}{ EMPIDIDAE } \\
\hline Drapetis (Elaphropeza) sp. & $\mathrm{X}$ & & 4 \\
\hline \multicolumn{4}{|l|}{ MUSCIDAE } \\
\hline Brontaea debilis (Williston, 1896) & $\mathrm{X}$ & & 4 \\
\hline Limnophora sp. & & $\mathrm{X}$ & 17 \\
\hline \multicolumn{4}{|l|}{ OTITIDAE } \\
\hline Otitidae spp. & $\mathrm{X}$ & & 4 \\
\hline \multicolumn{4}{|l|}{ RICHARDIDAE } \\
\hline Richardidae spp. & $\mathrm{X}$ & & 4 \\
\hline \multicolumn{4}{|l|}{ SARCOPHAGIDAE } \\
\hline Sarcophagidae spp. & $\mathrm{X}$ & $\mathrm{X}$ & 4,17 \\
\hline \multicolumn{4}{|l|}{ STRATYOMYIDAE } \\
\hline Hoplitimyia cf. mutabilis (Fabricius 1787) & $\mathrm{X}$ & & 4 \\
\hline \multicolumn{4}{|l|}{ SYRPHIDAE } \\
\hline Copestylum spp. & & $\mathrm{X}$ & 17 \\
\hline Syrphidae spp. & & $\mathrm{X}$ & 17 \\
\hline Toxomerus sp. & & $\mathrm{X}$ & 17 \\
\hline \multicolumn{4}{|l|}{ TABANIDAE } \\
\hline Tabanidae sp. & $\mathrm{X}$ & & 4 \\
\hline \multicolumn{4}{|l|}{ TACHINIDAE } \\
\hline Dexiinae (Dexiini) sp. & $\mathrm{X}$ & & 4 \\
\hline Lixophaga sp. & $\mathrm{X}$ & & 4 \\
\hline Tachinidae Indeterminado & $\mathrm{X}$ & & 4 \\
\hline Trichopodini (complexo Acaulona) sp. & $\mathrm{X}$ & & 4 \\
\hline Trichopodini (complexo Trichopoda) sp. & $\mathrm{X}$ & & 4 \\
\hline \multicolumn{4}{|l|}{ TEPHRITIDAE } \\
\hline Tephritidae sp. & $\mathrm{X}$ & & 4 \\
\hline \multicolumn{4}{|l|}{ HYMENOPTERA, ACULEATA } \\
\hline \multicolumn{4}{|l|}{ AMPULICIDAE } \\
\hline Ampulicidae sp. & $\mathrm{X}$ & & 4 \\
\hline \multicolumn{4}{|l|}{ BRACONIDAE } \\
\hline Braconidae spp. & $\mathrm{X}$ & & 4 \\
\hline \multicolumn{4}{|l|}{ CHALCIDIDAE } \\
\hline Chalcididae spp. & $\mathrm{X}$ & & 4 \\
\hline \multicolumn{4}{|l|}{ CHRYSIDIDAE } \\
\hline Chrysididae sp. & & $\mathrm{X}$ & 16 \\
\hline CRABRONIDAE & & & \\
\hline Larrinae spp. & $\mathrm{X}$ & & 4 \\
\hline Trachypus petiolatus (Spinola, 1841) & & $\mathrm{X}$ & 16 \\
\hline EUCHARITIDAE & & & \\
\hline Eucharitidae spp. & $\mathrm{X}$ & & 4 \\
\hline NYSSONIDAE & & & \\
\hline Bicyrtes sp. & $\mathrm{X}$ & & 4 \\
\hline Nyssoninae spp. & $\mathrm{X}$ & & 4 \\
\hline Rubrica sp. & $\mathrm{X}$ & & 4 \\
\hline PHILANTIDAE & & & \\
\hline Philantinae sp. & $\mathrm{X}$ & & 4 \\
\hline POMPILIDAE & & & \\
\hline Pepsis spp. & $\mathrm{X}$ & & 4 \\
\hline SCOLIIDAE & & & \\
\hline
\end{tabular}


Tab. I. Cont.

\begin{tabular}{|c|c|c|c|}
\hline & Cerrado & Pantanal & Referências \\
\hline Scoliinae spp. & $\mathrm{X}$ & & 4 \\
\hline \multicolumn{4}{|l|}{ SPHECIDAE } \\
\hline Amnophilinae spp. & $X$ & & 4 \\
\hline Eremnophila sp. & $\mathrm{X}$ & $\mathrm{X}$ & 4,16 \\
\hline Philanthus sp. & $\mathrm{X}$ & & 4 \\
\hline Sphecinae spp. & $\mathrm{X}$ & & 4 \\
\hline Sphex sp. & $\mathrm{X}$ & & 4 \\
\hline \multicolumn{4}{|l|}{ TIPHIIDAE } \\
\hline Tiphiidae spp. & $\mathrm{X}$ & & 4 \\
\hline \multicolumn{4}{|l|}{ VESPIDAE } \\
\hline Agelaia multipicta (Haliday, 1936) & & $\mathrm{X}$ & 16 \\
\hline Agelaia sp. & $\mathrm{X}$ & & 4 \\
\hline Ancistroceroides rafimaculus (Fox) & & $\mathrm{X}$ & 16 \\
\hline Brachygastra fistulosa Naumann, 1968 & $\mathrm{X}$ & & 4 \\
\hline Brachygastra lecheguana Latreille, 1824 & & $\mathrm{X}$ & 13 \\
\hline Brachygastra moulae Richards, 1978 & & $\mathrm{X}$ & 16 \\
\hline Brachygastra sp. & $\mathrm{X}$ & $\mathrm{X}$ & 4,16 \\
\hline Cyphomenes infernalis (Saussure, 1875) & & $\mathrm{X}$ & 16 \\
\hline Minixi suffusion (Fox) & & $\mathrm{X}$ & 16 \\
\hline Mischocyttarus sp. & $\mathrm{X}$ & $\mathrm{X}$ & 4,17 \\
\hline Nectarina sp. & $\mathrm{X}$ & & 4,6 \\
\hline Omicron tegulare (Fox, 1899) & & $\mathrm{X}$ & 16 \\
\hline Omicron tuberculatum (Fox, 1899) & & $\mathrm{X}$ & 16 \\
\hline Pachodynerus sp. & $\mathrm{X}$ & $\mathrm{X}$ & 4,16 \\
\hline Polistes canadensis (Linnaeus, 1758) & $\mathrm{X}$ & & 4 \\
\hline Polistes versicolor (Olivier,1791) & $\mathrm{X}$ & & 4 \\
\hline Polybia chrysothorax (Lichtenstein, 1796) & $\mathrm{X}$ & $\mathrm{X}$ & 4,16 \\
\hline Polybia ignobilis (Haliday, 1836) & $\mathrm{X}$ & $\mathrm{X}$ & $4,13,16$ \\
\hline Polybia paulista (Ihering, 1896) & & $\mathrm{X}$ & 13 \\
\hline Polybia sericea (Olivier, 1791) & & $\mathrm{X}$ & 13 \\
\hline Protonectarina sp. & $\mathrm{X}$ & & 4 \\
\hline Stenonartonia apicipennis (Fox, 1902) & & $\mathrm{X}$ & 16 \\
\hline Synoeca sp. & & $\mathrm{X}$ & 16 \\
\hline Synoeca surinama Linnaeus, 1767 & $\mathrm{X}$ & & 4 \\
\hline Zeta argillaceum (Linnaeus, 1758) & $\mathrm{X}$ & $\mathrm{X}$ & 4,16 \\
\hline Zethus schrottkyanus Ihering, 1911 & & $\mathrm{X}$ & 16 \\
\hline \multicolumn{4}{|l|}{ ORTHOPTERA } \\
\hline \multicolumn{4}{|l|}{ ACRIDIDAE } \\
\hline Acrididae 1 & $\mathrm{X}$ & & 4 \\
\hline \multicolumn{4}{|l|}{ BLATODEA } \\
\hline \multicolumn{4}{|l|}{ BLATTELIDAE } \\
\hline Blattelidae 1 & $\mathrm{X}$ & & 4 \\
\hline \multicolumn{4}{|l|}{ BLATTIDAE } \\
\hline Blattidae 1 & & $\mathrm{X}$ & 14 \\
\hline \multicolumn{4}{|l|}{ MANTODEA } \\
\hline Mantodea 1 & $\mathrm{X}$ & & 4 \\
\hline
\end{tabular}

comumente, ladrões de néctar: são pequenas, desprovidas de asas, possuem corpo liso, que limpam frequentemente; bem como liberam secreções antibióticas que supostamente reduzem a viabilidade do pólen. Entretanto, formigas podem ser importantes polinizadores de espécies que crescem junto ao solo, em ambientes áridos ou desérticos ou em altitudes elevadas (CORLETT, 2004).

Abelhas (Hymenoptera, Apoidea, Anthophila). As 155 espécies registradas pertencem a 68 gêneros e cinco famílias (MichenER, 2000), sendo Apidae a mais rica (109 espécies), seguida por Halictidae (25), Megachilidae (14), Colletidae (4) e Andrenidae (3). A riqueza de abelhas amostrada até o momento em MS é pequena se comparada à estimada, por exemplo, para o estado de São Paulo (SP) (729 spp.) (PEDro \& CAMARGo, 1999), onde são realizados levantamentos a mais de quatro décadas e onde ocorrem diversos grupos de pesquisas e acervos. Entretanto, trabalhos com coletas em sete localidades distintas no estado de SP (três na Mata Atlântica e quatro no cerrado), registraram total de 276 espécies e 88 gêneros, com Apidae como família mais 


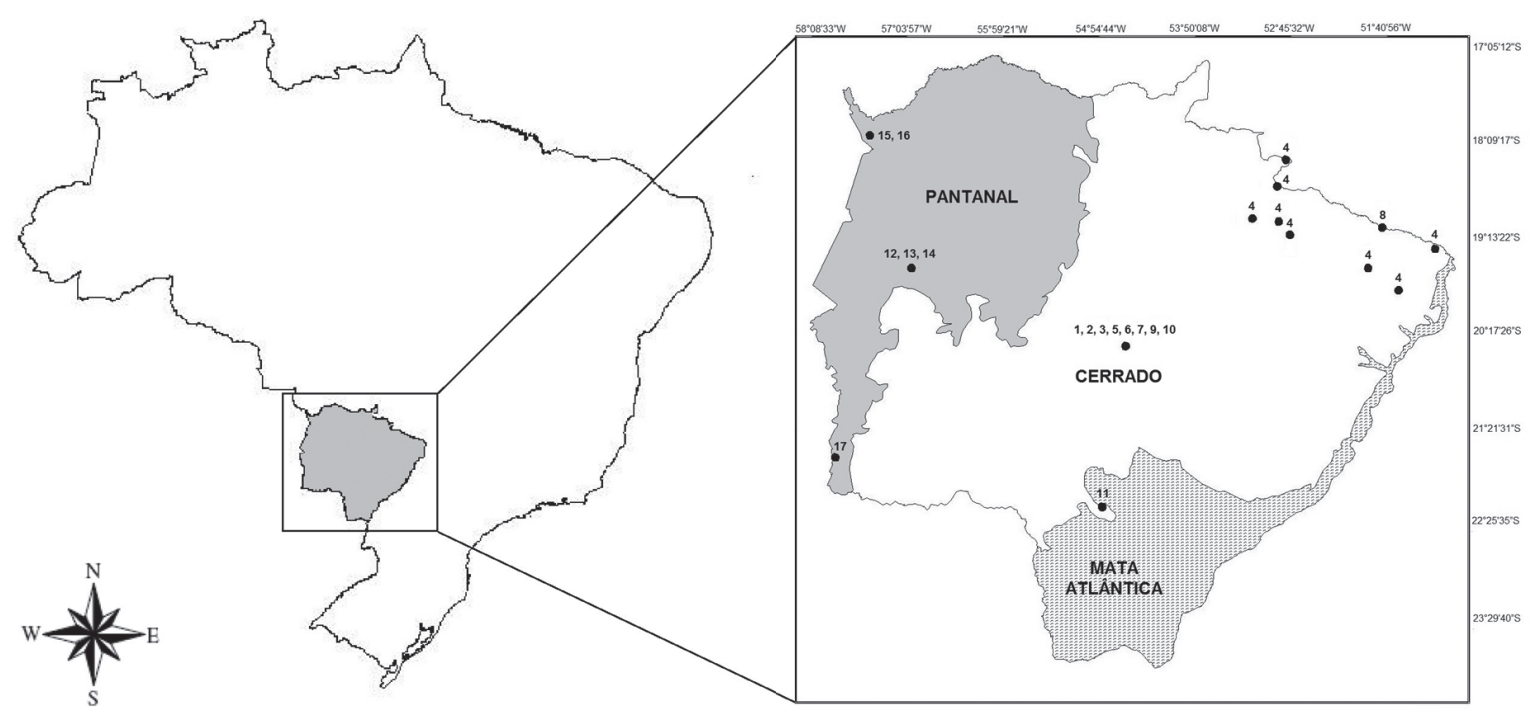

Fig. 1. Mapa com a distribuição dos estudos sobre visitantes florais no estado do Mato Grosso do Sul (1, Consolaro, 2002; 2, Rodrigues, 2002; 3, Oda, 2003; 4. Aoki \& Sigrist, 2006; 5. Oliveira et al., 2007; 6, Oliveira \& Sigrist, 2008; 7, Munin et al., 2008; 8, VieIra et al., 2008; 9. Maier, 2009; 10, Vilas Boas, 2009; 11, Ferreira et al., 2010; 12. Longo \& Fischer, 2006; 13, BofF, 2008; 14, Fava et al., 2011; 15, AoKi et al., 2012; 16, AuKo et al., 2012; 17, SouzA, 2012).

representativa, mas com 105 espécies registradas em Cerrado, valor ligeiramente menor que neste estudo (126 spp.).

Centris (13 spp.), Augochloropsis, Trigona (9 spp. cada) e Epicharis (7 spp.) foram os gêneros mais representativos (Tab. I) e estão entre os oito gêneros mais ricos das savanas tropicais (exceto Trigona) (PinHeIRoMachado et al., 2002). Apis melifera, exótica, foi a mais comum nos levantamentos, seguida pelas nativas Tetragonisca angustula, Trigona spinipes, Paratrigona lineata e Centris varia. Exceto $C$. varia, as demais espécies são consideradas generalistas, pois geralmente visitam amplo espectro de plantas (IMPERATRIZ-FONSECA et al., 2011).

Besouros (Coleoptera). Os 82 táxons de besouros com alguma identificação registrados para Mato Grosso do Sul estão relatados em apenas quatro estudos (AOKI \& SigRIST, 2006; Munin et al., 2008; FAVA et al., 2011; SouZA, 2012). Esta elevada riqueza de coleópteros mostra que flores são importantes sítios de coleta deste grupo de insetos. Dentre as 18 famílias registradas, Chrysomelidae foi a mais rica (20 espécies), seguida de Curculionidae (18) e Scarabaeidae (10). Representantes dessas famílias são comumente mencionados como visitantes florais e/ou polinizadores de diversas famílias de Angiospermas (GotTsberger, 1999; Corlett, 2004; LeNZI et al., 2005; CosTA \& Morais, 2008).

Borboletas/mariposas (Lepidoptera). As 53 espécies de lepidópteros registradas estão distribuídas em sete famílias, com destaque para Nymphalidae (16 espécies), Hesperiidae (16) e Lycaenidae (10), famílias com representantes de hábito diurno, período em que se concentrou a quase totalidade dos estudos realizados. No estado a amostragem de mariposas em flores praticamente inexiste, com duas espécies registradas em Bauhinia curvula por MuNin et al. (2008).
Formigas (Hymenoptera, Formicidae). Somente dois estudos contribuíram para o conhecimento da fauna de formigas em flores (AoKI \& SigRIST, 2006; SouZA, 2012), resultando em listagem de 23 espécies distribuídas em seis subfamílias, com destaque para Myrmicinae (7 espécies), Formicinae (6) e Dolichoderinae (4). Apesar de ocuparem todos os extratos de um ambiente, muitos grupos de formigas mantêm relações estreitas com plantas, obtendo recursos como pólen e néctar floral e extrafloral e, na maioria das vezes, protegendo a planta hospedeira de outros herbívoros (Oliveira et al., 1987).

Hemípteros (Hemiptera). Doze famílias foram listadas, e até o momento somente quatro, das 15 espécies registradas, foram identificadas. Representantes de Hemiptera são frequentemente amostrados em estudos que registram toda a fauna antófila, e geralmente visitam flores para comer partes florais, sendo o néctar de interesse secundário ou nulo (FAegri \& VAN DER PIJL, 1979).

Moscas/mosquitos (Diptera). Dentre as 34 espécies identificadas em algum nível taxonômico, foram registradas vinte famílias de moscas e mosquitos em visitas às flores, com destaque para Bombyliidae e Tachinidae em riqueza (5 spp. cada). No mundo, segundo Larson et al. (2001), Diptera corresponde à segunda ordem mais importante de insetos visitantes de flores, com pelo menos 71 famílias com representante antófilo.

Vespas (Hymenoptera, Apoidea, Aculeata). Treze famílias de vespas foram registradas em estudos de visitantes florais no estado, sendo que Vespidae foi majoritariamente a mais rica (26 spp.). Vespas são visitantes comuns em flores, embora algumas espécies sejam atraídas pela oportunidade 


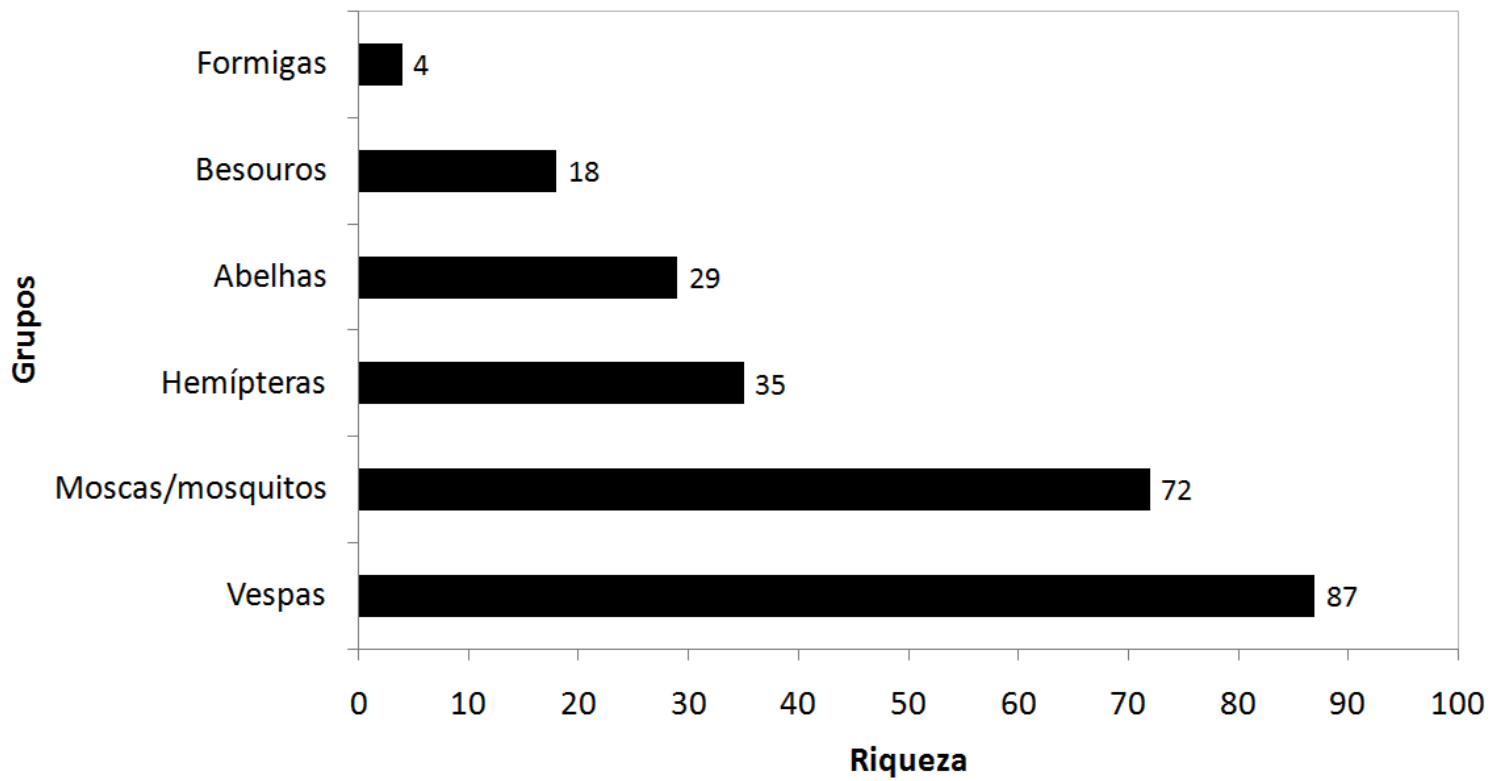

Fig. 2. Número mínimo de espécies não identificadas que constam em levantamentos realizados no Cerrado e Pantanal, Mato Grosso do Sul, Brasil.

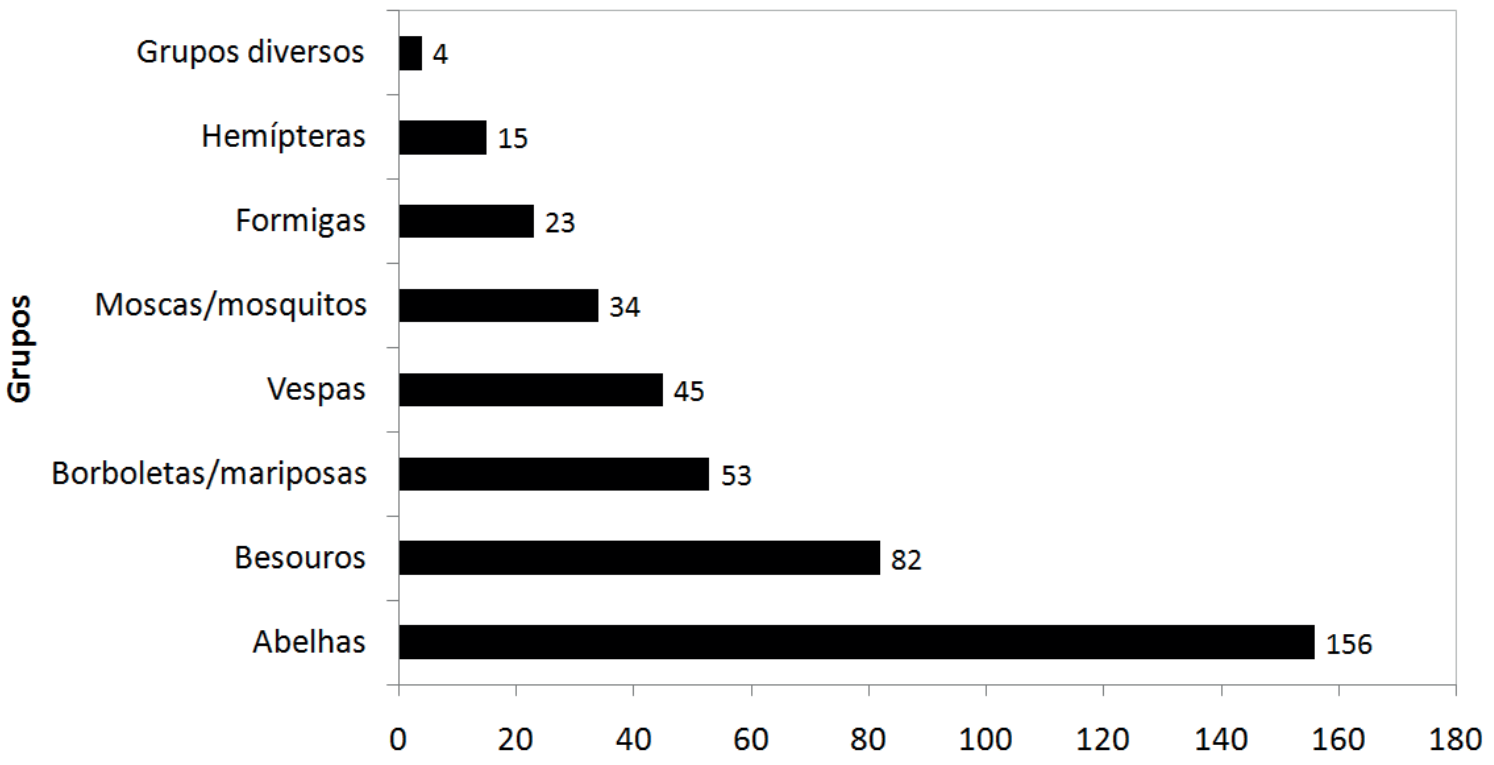

Fig. 3. Riqueza de insetos amostrados em flores em estudos realizados em vegetação de Cerrado e Pantanal, Mato Grosso do Sul, Brasil.

de predar outros visitantes (FAEGRI \& VAN DER PIJL, 1979; CORLETT, 2004).

Ordens diversas. Foram incluídos neste grupo representantes de Orthoptera (Acrididae) (grilos), Mantodea (louva-a-Deus) e Blattodae (Blattelidae e Blattidae) (baratas), que comumente são registrados em flores consumindo partes florais ou buscando alguma presa (Mantodea) e não atuando como polinizadores, com exceções (NAGAMitsu \& InOUE, 1997; Corlett, 2004; Vlasáková et al., 2008; Micheneau et al., 2010).

Principais acervos e grupos de pesquisa. No MS há somente duas instituições que abrigam espécimes de insetos que visitam flores: a Universidade Federal de Mato Grosso do Sul (UFMS) com a Coleção Zoológica de Referência da UFMS (ZUFMS) e a Universidade Federal da Grande Dourados (UFGD), com o Museu de Biodiversidade da Faculdade de Ciências Biológicas e Ambientais (Mubio/ UFGD). Temos apenas dois os grupos de pesquisas, ambos na UFMS, que trabalham direta ou indiretamente com insetos de visitantes de flores: (i) no campus Campo Grande, na linha de pesquisa "Conservação de Polinizadores" coordenado por Maria Rosângela Sigrist, (ii) no campus de Corumbá no grupo "Pesquisa em abelhas: biologia, diversidade e genética" coordenado por Aline Mackert dos Santos.

Principais dificuldades, lacunas de conhecimento e perspectivas para os próximos 10 anos. Para a maioria dos 
grupos amostrados (exceto abelhas, geralmente amostradas em flores) comparações foram limitadas por falta de dados e/ou estudos equivalentes no Brasil como um todo e não somente em MS. Dados sobre visitantes florais noturnos (e.g. besouros, mariposas, moscas) praticamente inexistem para o estado. Intercâmbio ou parceria entre profissionais que trabalham com levantamento de insetos em flores e taxonomistas destes grupos é incipiente, haja vista o elevado número de espécies na categoria de "indeterminado". Este fato é agravado pela falta de taxonomistas para boa parte dos grupos amostrados, necessidade de revisões taxonômicas e de boas coleções de referência no estado.

A dificuldade de identificação de material biológico é uma limitação tanto com relação à determinação da riqueza de espécies em determinado bioma, estado ou país, como para a comparação e utilização dos dados de interações relatadas nos trabalhos. No Brasil, para quase todos os grupos de insetos há escassez de especialistas para a identificação das espécies, portanto, são necessários incentivos ou ações que promovam a formação de taxonomistas e a integração entre os taxonomistas existentes e os centros de pesquisa de regiões com carência deste tipo de profissional, principalmente nas regiões Centro-Oeste e Norte.

Perspectivas e necessidades. Fomento das coleções a partir de novos levantamentos, mas com o fortalecimento dos acervos e grupos de pesquisa através de parcerias entre pesquisadores do estado e do Brasil, bem como formação de recursos humanos nestas áreas. Pesquisas com enfoque nas redes de interação, no papel dos visitantes florais na polinização das espécies vegetais, estudos sobre o efeito da sazonalidade na diversidade de visitantes florais principalmente no Pantanal em que esta é muito marcada, estudo dos efeitos da inclusão de espécies exóticas (como Apis mellifera) sobre as nativas de polinizadores são necessários.

Agradecimentos. CNPq, FUNDECT, Capes e Fundação Universidade Federal de Mato Grosso do Sul pelo apoio às nossas pesquisas; aos Ayr de Moura Bello, André V. L. Freitas, Carlos José Einicker Lamas, Gustavo Graciolli, Helcio Gil Santana e Rogério Silvestre, ao Manoel Fernando Demétrio, biólogo Daniel Máximo Corrêa de Alcântara pelo auxílio na identificação e/ou valiosos comentários sobre besouros, borboletas, hemípteros e moscas; Arnildo Pott pela revisão do abstract.

\section{REFERÊNCIAS BIBLIOGRÁFICAS}

Aoki, C. \& Sigrist, M. R. 2006. Inventário dos visitantes florais no Complexo Aporé-Sucuriú. In: Pagoto, T. C. S. \& DE Souza, P. R. orgs. Biodiversidade do Complexo Aporé-Sucuriú. Subsídios à conservação e ao manejo do Cerrado. Campo Grande, Editora da Universidade Federal de Mato Grosso do Sul, p.143-162.

Aoki, C.; Teixeira-Gamarra, M. C.; Barônio, G. J.; Sigrist, M. R. \& Laroca, S. 2012. Diversidade de abelhas (Hymenoptera: Apoidea) e recursos florais na RPPN Engenheiro Eliezer Batista, Pantanal de Mato Grosso do Sul. In: Rabelo, A. C. P.; Moreira, V. F.; Bertassoni, A. \& AOKI, C. orgs. Descobrindo o Paraíso. Aspectos Biológicos da Reserva Particular do Patrimônio Natural Engenheiro Eliezer Batista. Rio de Janeiro, Instituto Homem Pantaneiro, p.82-111.

Auko, T. H.; Trad, B. M.; Silvestre, R. \& Aoki, C. 2012. Vespas Aculeata da Reserva Particular do Patrimônio Natural Engenheiro Eliezer Batista In: Rabelo, A. C. P.; Moreira, V. F.; Bertassoni, A. \& Aoki, C. orgs. Descobrindo o Paraíso. Aspectos Biológicos da Reserva Particular do Patrimônio Natural Engenheiro Eliezer Batista. Rio de Janeiro, Instituto Homem Pantaneiro, p.240-261
Barbola, I. F.; Laroca, S. \& Alemida, M. C. 2000. Utilização de recursos florais por abelhas silvestres (Hymenoptera, Apoidea) da Floresta Estadual do Passa Dois (Lapa, Paraná, Brasil). Revista Brasileira de Entomologia 44:9-19.

BofF, S. 2008. Flora de capões e Hymenoptera (abelhas e vespas) visitantes de flores no pantanal do Miranda-Abobral. Dissertação de Mestrado. Campo Grande, Universidade Federal de Mato Grosso do Sul.

Consolaro, H. N. 2002. Levantamento das abelhas (Hymenoptera, Apoidea) do campus da Universidade Federal de Mato Grosso do Sul, Campo Grande, MS. Monografia de conclusão de curso. Campo Grande, Universidade Federal de Mato Grosso do Sul.

CORLETt, R. T. 2004. Flower visitors and pollination in the Oriental (Indomalayan) Region. Biological Reviews 79:497-532.

Costa, R. A. C. V. \& Morais, A. B. B. 2008. Fenologia e visitantes florais de Erythrina crista-galli (Leguminosae: Faboideae) em Santa Maria, Rio Grande do Sul, Brasil. Biotemas 21(2):51-56.

DuFÄ̈, M. \& ANSTETT, M. C. 2003. Conflicts between plants and pollinators that reproduce within inflorescences: evolutionary variations on a theme. Oikos 100:3-14.

FAEGRI, K. \& VAN DER PIJL, L. 1979. The principles of pollination ecology. London, Pergamon Press. 242p.

FaVA, W. S.; Sigrist, M. R. \& Covre, W. S. 2011. Attalea phalerata and Bactris glaucescens (Arecaceae, Arecoideae): Phenology and pollination ecology in the Pantanal, Brazil. Flora 206:575-584.

Ferreira, M. G.; Manente-Balestieri, F. C. D. \& Balestieri, J. B. P. 2010. Pólen coletado por Scaptotrigona depilis (Moure) (Hymenoptera, Meliponini), na região de Dourados, Mato Grosso do Sul, Brasil. Revista Brasileira de Entomologia 54(2):258-262.

GotTsberger, G. 1999. Pollination and evolution in neotropical Annonaceae. Plant Species Biology 14:143-152.

IBGE. 2004. Instituto Brasileiro de Geografia e Estatística. Disponível em: $<$ www.ibge.gov.br>. Acessado em 08.08.2012.

ImPERATRIZ-FonSECA, V.; SARAIVA, A. M. \& GonÇALVES, L. 2007. A iniciativa brasileira de polinizadores e os avanços para a compreensão do papel dos polinizadores como produtores de serviços ambientais. Bioscience Journal 23:100-106.

Imperatriz-Fonseca, V. L.; Alves-dos-Santos, I.; Santos-Filho, P. S.; Engels, W.; Ramalho, M.; Wilms, W.; Aguilar, J. B. V.; PinheiroMachado, C. A.; Alves, D. A. \& Kleinert, A. M. P. 2011. Checklist of bees and honey plants from São Paulo State, Brazil. Biota Neotropica 11(1a). Disponível em: <http://www.biotaneotropica.org.br/v11n1a/pt/ abstract?article+bn0321101a2011>.

LaRoCA, S. \& ORTH, A. I. 2002 Melissocoenology: historical perspective, method of sampling, and recommendations to the "Program of conservation and sustainable use of pollinators, with emphasis on bees" (ONU). In: Kevan, P. \& Imperatriz Fonseca, V. L. eds. Pollinating bees. The conservation link between agriculture and nature. Brasília, Ministério do Meio Ambiente, p.217-225.

Larson, B. M. H.; Kevan, P. G. \& InOuYe, D. W. 2001. Flies and flowers: The taxonomic diversity of anthophiles and pollinators. The Canadian Entomologist 133(4):439-465.

LenZi, M.; OrTH, A. I. \& Guerra, T. M. 2005. Ecologia da polinização de Momordica charantia L. (Cucurbitaceae), em Florianópolis, SC, Brasil. Revista Brasileira de Botânica 28:505-513.

LongO, J. M. \& FisCHER, E. 2006. Efeito da taxa de secreção de néctar sobre a polinização e a produção de sementes em flores de Passiflora speciosa Gardn. (Passifloraceae) no Pantanal. Revista Brasileira de Botânica 29:481-488.

MAIER, J. E. 2009. Levantamento de abelhas e atuação na polinização de espécies vegetais em remanescente urbano de cerrado, Campo Grande, Mato Grosso do Sul. Monografia de conclusão de curso. Campo Grande, Universidade Federal de Mato Grosso do Sul.

Micheneau, C.; Fournel, J.; Warren, B. H.; Hugel, S.; Gauvin-Bialecki, A.; Pailler, T.; Strasberg, D. \& Chase, M. W. 2010. Orthoptera, a new order of pollinator. Annals of Botany 105:1-10.

MichenER, C. D. 2000. The bees of the world. Baltimore, Maryland, Johns Hopkins University Press. 992p.

MMA - Ministério do Meio Ambiente. 2006. Disponível em: <http:// www.mma.gov.br/estruturas/chm/_arquivos/lista_polinizadores.pdf $>$. Acessado em: 08.08.2012. 
Munin, R. L.; Teixeira, R. C. \& Sigrist, M. R. 2008. Esfingofilia e sistema de reprodução de Bauhinia curvula Benth. (Leguminosae: Caesalpinioideae) em cerrado no Centro-Oeste brasileiro. Revista Brasileira de Botânica 31:15-25.

Nagamitsu, T. \& InOUE, T. 1997. Cockroach pollination and breeding system of Uvaria elmeri (Annonaceae) in a lowland mixed-Dipterocarp forest in Sarawak. American Journal of Botany 84:208-213.

Nyhagen, D. F.; Kragelund, C.; Olesen, J. M. \& Jones, C. G. 2001 Insular interactions between lizards and flowers: flower visitation by an endemic Mauritian gecko. Journal of Tropical Ecology 17:755-761.

ODA, F. H. 2003. Levantamento e o papel das abelhas nas flores de espécies vegetais em remanescente de cerrado em Campo Grande, MS. Monografia de conclusão de curso. Campo Grande, Universidade Federal de Mato Grosso do Sul.

OliveIRA, M. B. \& SigRist, M. R. 2008. Fenologia reprodutiva, polinização e reprodução de Dipteryx alata Vogel (Leguminosae - Papilionoideae) em Mato Grosso do Sul, Brasil. Revista Brasileira de Botânica 31:195-207.

Oliveira, M. I. B.; Polido, C. A.; Costa, L. C. \& Fava, W. S. 2007. Sistema reprodutivo e polinização de Byrsonima intermedia A. Juss. (Malpighiaceae) em Mato Grosso do Sul, Brasil. Revista Brasileira de Biociências 5:756-758

Oliveira, P. S.; Silva, A. F. \& Martins, A. B.1987. Ant foraging on extrafloral nectaries of Qualea grandiflora (Vochysiacea) in Cerrado vegetation: ants a potential antiherbivore agent. Oecologia 74:228-230.

Pedro, S. R. M. \& Camargo, J. M. F. 1999. Apoidea, Apiformes. In: Joly, C. A.; Bicudo, C. E. M.; Brandẽo, C. R. F. \& Cancello, E. M. eds. Biodiversidade do Estado de São Paulo, Brasil. Síntese do conhecimento ao final do século XX. Invertebrados Terrestres. São Paulo, FAPESP. vol. 5, p.193-211.

Pinheiro-Machado, C.; Santos, I. A.; Imperatriz-Fonseca, V. L.; Kleinert, A. M. P.; Silveira, F. A. 2002. Brazilian bee surveys: State of knowledge, conservation and sustainable use. In: KEvAN, P. \&
Imperatriz Fonseca, V. L. eds. Pollinating bees. The conservation link between agriculture and nature. Brasília, Ministério do Meio Ambiente, p. 115-129.

Proctor, M.; Yeo, P. \& LACK, A. 1996. The natural history of pollination. Portland, Timber Press. $487 \mathrm{p}$.

Rodrigues, L. C. 2002. Biologia da polinização de duas espécies de Bromelia (Bromeliaceae) em cerrado de Mato Grosso do Sul. Monografia de conclusão de curso. Campo Grande, Universidade Federal de Mato Grosso do Sul.

Sazima, M.; Vogel, S.; Prado, A. L.; Oliveira, D. M.; Franz, G. \& SAZIMA, I. 2001. The sweet jelly of Combretum lanceolatum flowers (Combretaceae): a cornucopia resource for bird pollinators in the Pantanal, western Brazil. Plant Systematics and Evolution 227:195208.

SouzA, C. S. 2012. Fauna antófila diurna em formação chaquenha brasileira: composição e flora visitada. Monografia de conclusão de curso. Campo Grande, Universidade Federal de Mato Grosso do Sul.

Simpson, B. B. \& NeFF, J. L. 1981. Floral rewards: alternatives to pollen and nectar. Annals of the Missouri Botanical Garden 68:301-322.

Stehlmann, J. R. \& Semir, J. 2001. Biologia reprodutiva de Calibrachoa elegans (Miers) (Solanaceae). Revista Brasileira de Botânica 24:43-49.

Vieira, G. H. C.; Marchini, L. C.; Souza, B. A. \& Moreti, A. C. C. C. 2008. Fontes florais usadas por abelhas (Hymenoptera, Apoidea) em área de Cerrado no município de Cassilândia, Mato Grosso do Sul, Brasil. Ciência e Agrotecnologia 32:1454-1460.

VILAS BoAs, J. C. 2009. Fenologia e biologia reprodutiva de Byrsonima intermedia A. Juss. e B. pachyphylla Griseb (Malpighiaceae) em remanescente de cerrado, Mato Grosso do Sul, Brasil. Dissertação de Mestrado. Campo Grande, Universidade Federal de Mato Grosso do Sul.

Vlasáková, B.; Kalinová, B.; Gustafsson, M. H. G. \& Teichert, H. 2008. Cockroaches as pollinators of Clusia aff. sellowiana (Clusiaceae) on inselbergs in French Guiana. Annals of Botany 102:295-304. 